\title{
Why lesbians should be encouraged to have regular cervical screening
}

\author{
Hazel J Henderson
}

\begin{abstract}
Introduction The UK National Health Service Cervical Screening Programme (NHSCSP) advice on cervical cytology screening states that women who have never had sex with men are at very low risk of developing cervical cancer, and advice regarding need for screening in lesbians is inconsistent.
\end{abstract}

Methods Literature review searching PubMed, Web of Science and the Internet for articles on lesbians, cervical cancer and cervical cancer risk factors focusing on human papillomavirus (HPV) and screening behaviours.

Results Case reports and prevalence studies show that HPV can be transmitted sexually between women. It is not known whether prevalence of HPV or cervical cancer differs between lesbians and heterosexual women. The evidence consistently shows that prevalence of non-

\section{Introduction}

Concerns have been raised that despite being at risk of cervical cancer, lesbians are under-utilising cervical screening services..$^{1-5}$ Lesbians are less preventionoriented in their health care behaviour, tend to avoid routine health care and are more likely to avoid screening tests such as Papanicolaou (Pap) smears. 1,3,6,7 Health inequalities may exist for lesbians due to lack of understanding of the health care needs of this group, on the part of the women themselves and health care services.

Lesbians and health care providers are sometimes unsure of whether cervical screening is necessary, particularly for women who have never been or who are no longer sexually active with men. ${ }^{8-11}$ Although human papillomavirus (HPV) infection, a causative factor in cervical cancer, can be transmitted sexually between women, the general belief is that cervical cancer is associated with heterosexual intercourse, and advice is still aimed predominantly at heterosexual women or women who have sex with men. Whether lesbians are at greater, similar or lower risk of cervical cancer compared to heterosexual women is contested. However, nonattendance for screening would put this group at higher risk of late diagnosis of cervical cancer, where prognosis is likely to be poorer.

\section{The lesbian population}

There is no universally agreed definition of the word 'lesbian', and it is not the aim of this review to fully capture the complexities of sexual orientation. Sexual orientation can incorporate different dimensions such as behaviour, desire or attraction, and identity. ${ }^{12}$ It is a common misconception that lesbians have only ever had sex with

Public Health Department, Croydon Primary Care Trust, Croydon, UK

Hazel J Henderson, MA, MFPH, Specialist Trainee in Public Health

Correspondence to: Ms Hazel Henderson, Public Health Department, Croydon Primary Care Trust, 13th Floor, Leon House, 233 High Street, Croydon CR9 1XT, UK.

E-mail: hazel.henderson@croydonpct.nhs.uk attendance for cervical screening is much higher in lesbian than heterosexual women, which is linked to a belief that lesbians are less susceptible to cervical cancer and have less need for screening. Despite sharing most of the same risk factors as heterosexual women, lesbians are much less likely to undergo regular screening.

Conclusions The NHSCSP should take a clear and consistent stance on the need for cervical screening in lesbians. Both the health care and the lesbian communities must be made aware of the fact that regular cervical screening is as important in this group as it is in the heterosexual female population.

Keywords cervical cancer, cervical screening, human papillomavirus, lesbian, screening policy

J Fam Plann Reprod Health Care 2009; 35(1): 49-52 (Accepted 19 August 2008)

\section{Key message points}

- Lesbians' cervical cancer risk factor profiles are not dissimilar to those of heterosexual women, with the exception that lower screening uptake in lesbians places them at greater risk of late diagnosis.

- Human papillomavirus can be sexually transmitted between women and it is unclear whether prevalence of cervical cancer is different in lesbians compared to heterosexual women.

- UK National Health Service Cervical Screening Programme policy should be changed to help ensure that a clear and consistent message is communicated regarding the need for cervical cytology screening in lesbians.

women. Many lesbians have had previous (and sometimes current) sexual relationships with men, whereas others have only ever had sex with women. Lesbians are an extremely diverse group comprising women from different age, ethnic, religious, socioeconomic and educational backgrounds. The word 'lesbian' is used by women to selfidentify according to a combination of the above dimensions. Sexual orientation is fluid and can vary across time, a fact that is often misunderstood.

At present there are very few reliable data available on the size of the UK's lesbian population. ${ }^{13}$ The true size of the lesbian population may be hidden due to stigmatisation and fear of discrimination. The most accurate estimate available suggests that $5-7 \%$ of the UK adult population identify themselves as lesbian, gay or bisexual. ${ }^{13}$

\section{Current NHSCSP policy regarding lesbians}

The top two risk factors for cervical cancer are HPV and non-attendance for regular cervical screening. Other risk factors such as smoking co-modify the risk of cervical cancer among HPV-positive women.

The UK National Health Service Cervical Screening Programme (NHSCSP) screening policy does not provide consistent advice for lesbians. The policy is consistent and clear for women aged 25-64 years who have sex with men, or who have a history of heterosexual intercourse. The policy regarding women who are exclusively lesbian (women who have never been sexually active with a man 
but have been sexually active with women) differs, as can be seen from the following NCSP recommendation: 14

"If a woman has never been sexually active with a man, then the research evidence shows that her chance of developing cervical cancer is very low indeed. In these circumstances a woman might choose to decline the invitation for cervical screening on this occasion. If a woman is not currently sexually active but has had male partners in the past, then we would recommend that she continues screening."

A key objective of this review is to explore the evidence base for the premise that women who are exclusively lesbian have a very low chance of developing cervical cancer.

\section{Methods}

The search terms 'lesbian AND cervical screening', 'women who have sex with women AND cervical screening', 'cervical cancer risk perception AND lesbians', 'cervical cancer risk AND lesbians' and 'screening attitudes in lesbians' were put into PubMed, Web of Science, and an Internet search engine (Google $\left.{ }^{\mathrm{TM}}\right)$. The 'related links' function was used to identify any articles missed during the initial search using PubMed. The reference sections of each article were used to identify further articles.

\section{Results}

\section{Cervical cancer risk in lesbians}

Some clinicians and lesbians still believe that lesbians do not need regular cervical screening, ${ }^{8-11}$ possibly based on the beliefs that lesbians never have sex with men and women do not transmit HPV to each other. Approximately four-fifths of lesbians have had sex with men. $8,9,15,16$ Evidence from case reports and prevalence studies of women who have only ever had sex with women shows that HPV can be transmitted sexually via female-to-female sexual activity.

Ferris et al. (1996) and O'Hanlan and Crum (1996) published case reports on two women who were exclusively lesbian but who had developed cervical intraepithelial neoplasia (CIN2). ${ }^{15,17}$ Although one woman had never had sex with men, her female partners had. Sexual activity reported by these women included active and passive oral sex, digital stimulation of clitoris and vagina, digital anal contact, and sharing of sex toys. Possible transmission routes may include oral-genital, as HPV has been known to colonise the oropharynx of some people with genital warts. ${ }^{18}$ Genital manipulation is another potential route. ${ }^{17}$ HPV DNA has previously been detected on fomites including rubber gloves and speculae, so it is feasible that it could be transmitted via shared sex toys. ${ }^{15}$

Marrazzo et al. examined HPV prevalence in 149 lesbians. ${ }^{19}$ Although presence of HPV infection was strongly associated with sexual history with men, the virus was present in women with no history of sex with men. Prevalence of HPV DNA in subjects who were exclusively lesbian was $19 \%$. Another study explored prevalence of genital HPV among 248 women who had sex with women (defined as 'having sex with a woman during the past year') using polymerase chain reaction techniques to detect HPV DNA..$^{8}$ Prevalence of genital HPV was $13 \%$. Further analysis found that $74 \%$ of the HPV infections detected were oncogenic types. HPV-associated squamous intraepithelial lesions were confirmed in some women who were exclusively lesbian. Other studies have found risk of sexually transmitted infections (STIs) in lesbians (including HPV) to be linked to increased sexual exposures with women. ${ }^{20}$
Prevalence of cervical dysplasia and cancer in lesbians

Bailey et al. explored the prevalence of cervical dysplasia in a sample of 606 lesbians attending lesbian sexual health clinics in London using self-completed questionnaires. ${ }^{21}$ Cervical cytology results were confirmed using patient records. One-fifth of the women studied were exclusively lesbian, which is consistent with the proportions found in other studies. $8,9,15,16$ Cervical dysplasia was present in $3.3 \%$ of participants. In the exclusively lesbian group $1.7 \%$ had cervical dysplasia, compared to $3.7 \%$ of those who had a history of sex with men. This difference was not statistically significant. The lesbians in this study were at some risk of cervical cancer due to the presence of risk factors including early age at first intercourse, large number of sexual partners, smoking, and presence of cervical dysplasia consistent with HPV infection.

Gatson Grindel et al. examined cancer prevention and screening behaviours in 1139 lesbians in a USA nationwide study. ${ }^{22}$ A quarter of lesbians failed to meet the national screening guidelines and $79.6 \%$ of the sample reported having had an abnormal smear in the past.

Valanis et al. compared prevalence of cervical cancer in women with different sexual orientations, as part of a large USA cohort study of 93311 post-menopausal women aged 50-79 years. ${ }^{4}$ A key finding was that age-standardised prevalence of cervical cancer was highest in lifetime lesbians $(2.2 \%)$ compared to heterosexual women $(1.3 \%)$.

\section{Cervical screening uptake in lesbians}

It could be assumed that lesbians are less likely to undergo regular cervical screening than heterosexual women because messages on the need for cervical screening in this group can be inconsistent or non-existent.

In 2001, Marrazzo et al. studied cervical screening practices in a sample of 248 women who had sex with women in Seattle. ${ }^{8}$ This study found screening practices among women who had sex with women to be closely linked to previous or current sex with men. Women who were exclusively lesbian were significantly different from women who had a sexual history with men, in that their first Pap smear occurred at an older age, they had fewer smears in the last 5 years, and had a longer interval between their two most recent smears. The most commonly reported reasons were prior negative experiences with Pap tests, and a belief that smears are not necessary unless heterosexually active. Some $10 \%$ of the study participants had been informed by a physician that they did not need a Pap test since they were not heterosexually active.

Matthews et al. (2004) compared cervical screening patterns in a sample of 550 lesbians and 279 heterosexual women. ${ }^{23}$ Compared to their heterosexual counterparts, lesbians were significantly less likely to attend for regular Pap smears. Factors that independently predicted regular screening were abnormal smear history, being heterosexual and annual medical visits. These findings are consistent with other studies that have found disparities in cervical screening rates based on sexual orientation. ${ }^{1,4,16}$ These disparities are possibly due to lesbians having to contend with more barriers to screening. Barriers may include lack of knowledge of lesbians' screening needs, fear of discrimination within the health care setting and mistrust of health care providers.

Rankow and Tessaro (1998) explored prevalence of cervical cancer risk factors and screening behaviours in a sample of 576 lesbians and women who had sex with women. ${ }^{16}$ Self-reported smoking prevalence was $4 \%$ higher than the state average for women. Thirty five percent of the women reported having had 1-3 male partners and $28 \%$ 
reported 4-10 previous male partners. Half of the participants had their first sexual experience with a man before reaching the age of 18 years, and $30 \%$ of all participants had a history of STIs. Despite having risk factors for cervical cancer, a quarter of the participants had not had a Pap test in the past 3 years, with women who reported positive experiences of health care providers being more likely to have ever had a Pap test. Forty three percent of the participants reported undergoing a Pap test in the past year compared to an average of $67 \%$ for the state (screening guidelines in the USA at the time recommended yearly Pap tests). Once age, education and income were controlled for, women who reported past discrimination within the health care system were much less likely to have had a Pap test.

Cochran et al. (2001) published a meta-analysis examining cervical cancer risk factors and screening behaviours among lesbian and bisexual women in the USA. ${ }^{3}$ The study combined data from seven studies that met the inclusion criteria, giving a total sample size of 11876 lesbians and women who had sex with women, and compared findings to national estimates for women. The key finding was that risk factors (including smoking and non-attendance for screening) for gynaecological cancers (including cervical) are more common in the lesbian population.

In a large cohort study of post-menopausal women, Valanis et al. found that women who had never had a Pap smear were 2.33 times more likely to be lesbian or bisexual $(p<0.01) .{ }^{4}$ Participants who had not had a smear in over 3 years were 1.69 times more likely to be lesbian or bisexual $(p<0.001)$.

\section{Beliefs about cervical cancer risk in lesbians}

The Health Belief Model may explain cervical screening behaviour in lesbians. 9 This model proposes that when making decisions about whether to undertake a given health behaviour, people will weigh the costs against the benefits, and that this decision-making process is affected by perceived susceptibility, severity, barriers and benefits. The potential of this model to explain lesbians' screening behaviour was explored by Price et al.

Price et al. (1996) studied 330 women of differing sexual orientations. 9 The key difference between lesbians and heterosexual women was that lesbians perceived themselves to be significantly less susceptible to cervical cancer, although they had the same understanding of the severity of cervical cancer and benefits of screening as did other women. The study also tested women's knowledge of the risk factors for cervical cancer. The only difference between the groups was that lesbians were significantly more likely to report 'sex with a man' as a risk factor compared to bisexual or heterosexual women. The finding that lesbians perceived their risk to be less than that of heterosexual women is interesting, given that this study found no difference between the various groups in terms of risk factors.

In 2005, Fish and Anthony conducted a national survey of health behaviour in 1066 lesbians in the UK. ${ }^{1}$ Fifty seven percent of lesbians perceived their risk of cervical cancer to be less than that of heterosexual women, $12 \%$ had never had a Pap smear and $15 \%$ no longer attended for screening although they were still eligible. Some $27 \%$ of lesbians were not attending for screening compared to $15 \%$ of the UK female population. Women who perceived themselves to be at no risk were more likely to have never attended for screening. It has been argued that nonattendance at screening may be linked to previous negative experiences. ${ }^{3,5-7}$ This study did not support this theory but did find a correlation between good screening experiences and future re-attendance. ${ }^{1}$

\section{Discussion}

There are inherent problems in researching lesbian populations. The first problem is that the lesbian population is difficult to identify and enumerate, so there is no sampling frame. Most studies have recruited small numbers and are prone to sampling bias, meaning that the results may not be generalisable to the whole lesbian population. Like any other population, lesbians are a very diverse group comprising women from a wide range of backgrounds. Many studies stated that highly educated women with high incomes were over-represented, and these are the women who are most likely to undergo regular cervical screening. ${ }^{23}$ These studies may therefore underestimate the extent of the problem.

Some studies compared lesbians to heterosexual women in terms of screening behaviour, risk factors and HPV prevalence. $1,3,4,9,16,23$ This provided a means of comparison if matched on relevant variables such as age. Some studies consisted only of lesbians or women who had sex with women, and compared the results with existing national and local data on the female population. This is potentially problematic if different methods of sampling and recruitment were used.

Self-reporting, relying on honest and accurate recall of events, was often used to establish screening practices and risk factors. The finding that lesbians are less likely to undergo regular smears is consistent across all studies, so it is unlikely that recall bias occurred in any systematic way that differed between lesbians and heterosexual women. Some studies used anonymous self-reporting, which may have helped improve the accuracy of reporting on some items, such as number of previous sexual partners.

Small sample sizes were a problem in some studies, although some attempted to overcome this problem by using large cohorts or meta-analysis.

Most of the studies discussed were from the USA, where cervical screening policy differs from the UK. In the USA, referral for screening is often via a physician and may be undertaken opportunistically during routine family planning visits. This is very different to the UK, where all eligible women registered with a general practitioner are invited for screening either every 3 or 5 years (depending on age).

It would be useful to undertake HPV DNA typing of lesbians and their sexual partners to explore how HPV is sexually transmitted between women, and how efficient transmission is.

The current evidence base does not support the NHSCSP's premise that women who are exclusively lesbian have a low risk of developing cervical cancer. This advice may contribute to non-attendance for screening, meaning that cervical dysplasia cannot be detected until symptoms appear, by which time treatment is likely to be less effective and the prognosis poorer. The NHSCSP has recently tried to redress this by stating that although risk is low in lesbians, screening is advisable. However, the assertion that risk of cervical cancer is very low in lesbians is not evidence-based and should be changed to reflect the evidence.

Work needs to be undertaken to ensure that a clear and consistent message is communicated to lesbians, and to health professionals providing cervical screening, regarding the need to undergo regular screening (regardless of sexual history with men). Specific advice should be provided for lesbians to ensure that there is no ambiguity surrounding the need for cervical screening in this group, and to encourage increased uptake of this important and effective programme. 


\section{Acknowledgements}

The author would like to thank Dr Jean Chapple who helped to edit and proofread this article. She would also like to thank Sandra Mounier-Jack from the London School of Hygiene and Tropical Medicine who advised on the author's original MSc dissertation on this topic.

\section{Statements on funding and competing interests \\ Funding None identified.}

Competing interests None identified.

\section{References}

1 Fish J, Anthony D. UK national lesbians and health care survey. Women Health 2005; 41: 27-45.

2 Roberts SJ, Patsdaughter CA, Grindel CG, Tarmina MS. Health related behaviors and cancer screening of lesbians: results of the Boston Lesbian Health Project II. Women Health 2004; 39: $41-55$

3 Cochran SD, Mays VM, Bowen D, Gage S, Bybee D, Roberts $\mathrm{SJ}$, et al. Cancer-related risk indicators and preventive screening behaviours among lesbians and bisexual women. Am J Public Health 2001; 91: 591-597.

4 Valanis BG, Bowen DJ, Bassford T, Whitlock E, Charney P, Carter RA. Sexual orientation and health: comparisons in the Women's Health Initiative sample. Arch Fam Med 2000; 9: 843-853.

5 Lee R. Health care problems of lesbian, gay, bisexual, and transgender patients. West J Med 2000; 172: 403-408.

6 Bernhard LA. Lesbian health and health care. Annu Rev Nurs Res 2001; 19: 145-177.

7 McNair RP. Lesbian health inequalities: a cultural minority issue for health professionals. Med J Aust 2003; 178: 643-645.

8 Marrazzo JM, Koutsky LA, Kiviat NB, Kuypers JM, Stine K. Papanicolaou test screening and prevalence of genital human papillomavirus among women who have sex with women. Am J Public Health 2001; 91: 947-952.

9 Price JH, Easton AN, Telljohann SK, Wallace PB. Perceptions of cervical cancer and pap smear screening behavior by women's sexual orientation. J Community Health 1996; 21: 89-105.

10 White J, Levinson W. Primary care of lesbian patients. J Gen Intern Med 1993; 8: 41-47.
11 Fish J, Wilkinson S. Lesbians and cervical screening: preliminary results from a UK study of lesbian health. Psychology Women Section Review 2000; 2: 5-15.

12 Solarz AL. Lesbian Health: Current Assessment and Directions for the Future. Washington, DC: National Academy Press, 1999.

13 Final Regulatory Impact Assessment: Civil Partnership Act 2004. Department of Trade and Industry. http://www.berr. gov.uk/files/file23829.pdf [Accessed 3 June 2008].

14 NHS Cervical Screening Programme website. Section on "What about women who are not sexually active?" http://www.cancerscreening.nhs.uk/cervical/index.html\#eligible [Accessed 15 April 2008].

15 Ferris DG, Batish S, Wright TC, Cushing C, Scott EH. A neglected lesbian health concern: cervical neoplasia. J Fam Pract 1996; 43: 581-584.

16 Rankow EJ, Tessaro I. Cervical cancer risk and Papanicolaou screening in a sample of lesbian and bisexual women. J Fam Pract 1998; 47: 139-143.

17 O'Hanlan KA, Crum CP. Human papillomavirus-associated cervical intraepithelial neoplasia following lesbian sex. Obstet Gynecol 1996; 88: 702-703.

18 Clarke J, Terry RM, Lacey CJ. A study to estimate the prevalence of upper respiratory tract papillomatosis in patients with genital warts. Int J STD AIDS 1991; 2: 114-115.

19 Marrazzo JM, Koutsky LA, Stine KL, Kuypers JM, Grubert TA, Galloway DA, et al. Genital human papillomavirus infection in women who have sex with women. J Infect Dis 1998; 178: 1604-1609.

20 Bauer GR, Welles SL. Beyond assumptions of negligible risk: sexually transmitted diseases and women who have sex with women. Am J Public Health 2001; 91: 1282-1286.

21 Bailey JV, Kavanagh J, Owen C, McLean KA, Skinner CJ. Lesbians and cervical screening. Br J Gen Pract 2000; 50 481-482.

22 Gatson Grindel C, McGehee LA, Patsdaughter CA, Roberts SJ. Cancer prevention and screening behaviors and lesbians. Women Health 2006; 44: 15-39.

23 Matthews AK, Brandenburg DL, Johnson T, Hughes TL. Correlates of under-utilization of gynecological cancer screening among lesbian and heterosexual women. Prev Med 2004; 38: 105-113.

\section{JOURNAL REVIEW}

Trends in sexually transmitted infections (other than HIV) in older persons: analysis of data from an enhanced surveillance system. Bodley-Tickell AT, Olowokure B, Bhaduri S, White DJ, Ward D, Ross JD, et al. Sex Transm Infect 2008: 84: 312-317

There are few data concerning sexually transmitted infections (STIs) that are specific to those aged 45 years and over and this age group is often excluded from studies as researchers and policymakers focus on young people. This Health Protection Agency study in the West
Midlands compared STI attendances in different age groups and found that rates for five STIs (i.e. syphilis, chlamydia, gonorrhoea herpes and genital warts) in those aged over 45 years were significantly higher in 2003 compared to 1996 , and the cumulative rate of infection in this group more than doubled from 16.7 per 100000 in 1996 to 36.3 per 100000 in 2003. Also, the increase in rates was greater in those aged 45 years and over than in people aged less than 45 years. The authors suggest that these trends are likely to continue, and may increase over time as successive cohorts with more liberal sexual attitudes and behaviours age and as older people are increasingly likely to be single due to divorce or separation. The authors suggest that education programmes around STIs should cater for all age groups, not just the young.

Reviewed by Anne Szarewski, PhD, FFSRH Clinical Consultant and Honorary Senior Lecturer, Cancer Research UK Centre for Epidemiology, Mathematics and Statistics, Wolfson Institute of Preventive Medicine, London, UK

\section{NEWS ROUNDUP}

\section{Women's Health Concern Appeal}

Women's Health Concern is launching an appeal to raise funds for its free and confidential telephone health advice and personal e-mail response services, via specialist nurses. This helpline is for women who do not seek help from their general practitioner because of embarrassment or other reasons. These health problems can impact on their partner, family and ability to go to work. The charity offers help and advice to women on a wide variety of gynaecological, sexual health and reproductive issues, promoting knowledge and awareness of specific diseases and medical conditions and has a range of fact sheets to download from their website.

Source: www womens-health-concern.org

\section{Cervical Cancer Prevention Week 18-24 January 2009}

The European Cervical Cancer Association (ECCA) in partnership with the European Cancer Organisation, the Association of European Cancer Leagues and the International Union against Cancer are collecting signature for the STOP Cervical Cancer Petition (www.CervicalCancerPetition.eu). This calls on the European Commission and national governments to support the implementation of organised cervical cancer screening programmes across Europe. The objective of the petition is to obtain 1 million signatures and then use it to draw attention in the European Parliament to the issue of cervical cancer prevention. Although the UK screening programme has been very successful, the UK is among a small minority of countries that benefit from organised screening programmes. Every year in Europe 50000 women develop and 25000 women die from cervical cancer. Effective prevention programmes could prevent the vast majority of these cases; sign the petition and help to bring this fact to the attention of politicians across Europe.

Source: www.CervicalCancerPetition.eu

Reviewed by Henrietta Hughes, MRCGP, DFSRH General Practitioner, London, UK 